\title{
943 3D IN VITRO TUMOR MODEL PLATFORM RAPIDLY ASSAYS IMMUNOTHERAPY EFFICACY WITH HIGH CONTENT IMAGING AND DOWNSTREAM PHENOTYPIC CHANGES WITH FLOW CYTOMETRY AND CYTOKINE ANALYSIS
}

${ }^{1}$ Bin Xue, ${ }^{2}$ Julia Schuler*, ${ }^{1}$ Christopher Harrod, ${ }^{1}$ Kolin Hribar. ${ }^{1}$ Cypre Inc, San Francisco, CA, USA; ${ }^{2}$ Charles River Discovery Research Service, Freiburg, Germany

Background Cancer immunotherapy represents a burgeoning new direction for oncology therapeutic innovation, with the principal thesis of activating one's own immune system to irradicate cancer as opposed to the injection of foreign cytotoxic agents like chemotherapy. The first generation of checkpoint inhibitors unleash cytotoxic $\mathrm{T}$ cells to locate and kill their tumor target, however, only a small subset of patients (e.g. $\sim 30 \%$ of NSCLC patients ${ }^{1}$ ) respond favorably. In order to advance the next generation of immunotherapy to the clinic, we critically need models which more accurately represent the immunosuppressive tumor microenvironment (TME).

Methods Here, we describe a novel 3D in vitro tumor model platform which engineers the tumor, stromal, and immune cell compartments of the TME in an extracellular matrix hydrogel $\left(\right.$ VersaGel ${ }^{2}$ ) and a high throughput 96-well format. The system has been extensively tested across multiple solid tumor indications, such as colorectal, lung, pancreatic, breast, and others. Specifically for this study, we utilized NSCLC PDX cells from the Charles River compendium and cocultured with human dermal fibroblasts (HDF) and PBMCs to study the effects of checkpoint inhibitor monoclonal antibodies, Pembrolizumab (anti-PD1) and Atezolizumab (anti-PDL1), on T cell infiltration and $\mathrm{T}$ cell-mediated killing using high content imaging. Supernatants were analyzed for cytokines and the 3D models were subsequently digested for flow cytometry.

Results The 3D models demonstrated varying degrees of $\mathrm{T}$ cell infiltration and killing capacity across PDX in a dose-dependent manner to checkpoint inhibitors, and the inclusion of fibroblasts played a critical role in further modulating response. Moreover, the data revealed clinically-relevant levels of $\mathrm{CD} 3+, \mathrm{CD} 4+$, and $\mathrm{CD} 8+\mathrm{T}$ cell subpopulations and cytokine secretions such as IFN-gamma.

Conclusions These data suggest a novel 3D model platform for assaying immunotherapeutic efficacy as well as its mechanism of action in the context of the TME. Future studies will include applying this novel platform to additional tumor models and screening multiple forms of immunotherapy - such as small molecules, biologics, and cell and gene therapy - in drug discovery, preclinical testing, and precision medicine.

\section{REFERENCES}

1. Haslam A, Prasad V. Estimation of the percentage of US patients with cancer who are eligible for and respond to checkpoint inhibitor immunotherapy drugs. JAMA Netw 2019;2(5):e192535.

2. Hribar KC, Wheeler CJ, Bazarov A, Varshneya K, Yamada R, Buckley P, Patil CG. A simple three-dimensional hydrogel platform enables ex vivo cell culture of patient and PDX tumors for assaying their response to clinically relevant therapies. Mol Canc Ther 2019;18(3):718-725.

http://dx.doi.org/10.1136/jitc-2021-SITC2021.943 